\title{
XVI.
}

\section{Ergänzung der Berichtigung: des}

Herrn Dr. Urbantschitseh. A. f. O. Bd. X. S. 86.

Ich erlaube mir, die diesbeztigliche Notiz nach einer Richtung hin noch zu ergänzen. Ich finde nämlich in Prof. Herrmann M e yer's Lehrbuch der Anatomie des Menschen, und zwar bereits in der II. Auflage 1861 und in der III. Auflage 1873 eine Aufzählang der normal in der Paukenhöhle vorkommenden Sehleimhautduplicaturen, eine Angabe, die allen Autoren, die bisher über diesen Gegenstand geschrieben, und anch mir entgangen war. Nach M. kommen folgende Schleimhautduplicaturen vor:

1. das Lig. corpor, ineudis,

2. die hintere und vordere Tröltsch'sche Falte,

3. als grössere Falten, die der Chorda tympani, dex Sebne des Tensor tymp. und der Sehne des M. stapedius mit dem Steigbügel. - -

Es ist diose Angabe Meyer's, eines so gewiegten Anatomen vom Fache, zur Beurtheilung des normalen oder pathologischen Vorlkommens dieser Gebilde sicherlich nicht zu unterschätzen.

E. Zaufal.

\section{XVII.}

\section{Berichtigung.}

In dem letzten Artikel von E. Z a u fal "Geschichtliches über die normalen Bewegungen der Pharyngealmündung der Eustachi'schen Röhre" wird die Behauptung aufgestellt; dass ich einen pathologischen Fall mit Parese des hinteren Gaumenbogens, als "typiscb für die normalen Bewegungen beschrieben hätte."

Dieser Fall wird auf Seite 4 meines Aufsatzes erzählt und dabei, um einer diesbezüglichen Bemängelung $\mathrm{Z}$ a u fal's zu begegnen, führe ich es hier an, auch ausdrücklich erklärt: "ich sehe die erste Bewegung an der Insertionsstelle des Gaumensegels am unteren Ende der äusseren Wand der Choane (Plica salpingo-palat.)"

Auf Seite 3 aber steht:

Sehr oft konnte ich die hintere Hälfte der Tubenmündung mit dem Tubenwulste in seiner ganzen Ausdehnung, darüber das Dach 